\title{
The Dynamic Behavior of a Discrete Vertical and Horizontal Transmitted Disease Model under Constant Vaccination
}

\author{
Mingshan Li, Xiumin Liu, Xiaoliang Zhou* \\ School of Mathematics and Statistics, Lingnan Normal University, Zhanjiang, China \\ Email: *zxlmath@163.com
}

How to cite this paper: Li, M.S., Liu, X.M. and Zhou, X.L. (2016) The Dynamic Behavior of a Discrete Vertical and Horizontal Transmitted Disease Model under Constant Vaccination. International Journal of Modern Nonlinear Theory and Application, 5, 171-184.

http://dx.doi.org/10.4236/ijmnta.2016.54017

Received: October 16, 2016

Accepted: November 15, 2016

Published: November 18, 2016

Copyright $\odot 2016$ by authors and Scientific Research Publishing Inc. This work is licensed under the Creative Commons Attribution International License (CC BY 4.0).

http://creativecommons.org/licenses/by/4.0/

\begin{abstract}
In this paper, a class of discrete vertical and horizontal transmitted disease model under constant vaccination is researched. Under the hypothesis of population being constant size, the model is transformed into a planar map and its equilibrium points and the corresponding eigenvalues are solved out. By discussing the influence of coefficient parameters on the eigenvalues, the hyperbolicity of equilibrium points is determined. By getting the equations of flows on center manifold, the direction and stability of the transcritical bifurcation and flip bifurcation are discussed.
\end{abstract}

\section{Keywords}

Vertical and Horizontal Transmission, Vaccination, Center Manifold, Transcritical Bifurcation, Flip Bifurcation

\section{Introduction}

The SIR infections disease model is an important model and has been studied by many authors [1]-[8]. The basic and important research subjects for these systems are local and global stability of the disease-free equilibrium and the endemic equilibrium, existence of periodic solutions, persistence and extinction of the disease, etc. In recent years, the study of vaccination, treatment, and associated behavioral changes related to disease transmission has been the subject of intense theoretical analysis [4] [9] [10] [11] [12]. In 2008, Meng and Chen [13] considered a class of continuous vertical and horizontal transmitted epidemic model under constant vaccination

$$
\left\{\begin{array}{l}
\dot{S}=-\beta S I-b S+(1-m) p d I+(1-m) b(S+R), \\
\dot{I}=\beta S I-d I-r I+q d I, \\
\dot{R}=r I-b R+m p d I+m b(S+R)
\end{array}\right.
$$


where $S$ represents the proportion of individuals susceptible to the disease, who are born (with $b$ ) and die (with $d$ ) at the same rate $b(b=d)$ and have mean life expectancy $1 / b$. The susceptible become infectious at a bilinear rate $\beta I$, where $I$ is the proportion of infectious individuals and $\beta$ is the contact rate. The infectious recover (i.e. acquire lifelong immunity) at a rate $r$, so that $1 / r$ is the mean infectious period. The constant $p, q, 0<p<1,0<q<1$, and $p+q=1$, where $p$ is the proportion of the offspring of infective parents that are susceptible individuals, and $q$ is the proportion of the offspring of infective parents that are infective individuals. In their work, the basic reproductive rate determining the stability of disease-free equilibrium point and endemic equilibrium point was found out and the local and global stability of the equilibrium points have been researched by using Lyapunov function and Dulac function.

Due to a lot of discrete-time models are not trivial analogues of their continuous ones and simple discrete-time models can even exhibit complex behavior (see [14]), in this paper, we pay attention to the discrete situation of Equation (1) as follows

$$
\left\{\begin{array}{l}
S_{n+1}=-\beta S_{n} I_{n}+(1-m b) S_{n}+(1-m) p d I_{n}+(1-m) b R_{n}, \\
I_{n+1}=\beta S_{n} I_{n}+(1-d-r) I_{n}+q d I_{n}, \\
R_{n+1}=r I_{n}+(1-b) R_{n}+m p d I_{n}+m b\left(S_{n}+R_{n}\right)
\end{array}\right.
$$

where $S_{n}, I_{n}$ and $R_{n}$ represent susceptible, infective and recovered subgroups, $n$ represent a fixed time. Under the hypothesis of population being constant size, the model is transformed into a planar map and its equilibrium points and the corresponding eigenvalues are solved out. By discussing the influence of coefficient parameters on the eigenvalues, we determine the hyperbolicity of equilibrium points. Further, we get the equations of flows on center manifold and discuss the direction and stability of the transcritical bifurcation and flip bifurcation.

\section{Hyperbolic and Non-Hyperbolic Cases}

In this section, we will discuss the hyperbolic and non-hyperbolic cases in a two parameters space parameter. In view of assumption that population is a constant size, i.e.,

$$
S_{n}+I_{n}+R_{n}=1
$$

system Equation (2) can be changed into

$$
\left\{\begin{array}{l}
S_{n+1}=-\beta S_{n} I_{n}+(1-b) S_{n}-(1-m)(b-p d) I_{n}+(1-m) b, \\
I_{n+1}=\beta S_{n} I_{n}+(1-p d-r) I_{n} .
\end{array}\right.
$$

Rewrite Equation (4) as a planar map $F$ :

$$
\left[\begin{array}{l}
S \\
I
\end{array}\right] \rightarrow\left[\begin{array}{c}
-\beta S I+(1-b) S-(1-m)(b-p d) I+(1-m) b \\
\beta S I+(1-p d-r) I
\end{array}\right]
$$

It is obvious that this map has a disease-free equilibrium point $P(1-m, 0)$ and an endemic equilibrium point $Q\left(S^{*}, I^{*}\right)$ where

$$
S^{*}=\frac{p d+r}{\beta}, \quad I^{*}=\frac{(1-m) b \beta}{\beta[(1-m) b+r+m p d]}, \quad k_{1}=\frac{(1-m) \beta}{p d+r} \text {. }
$$


Theorem 1. The equilibrium point $P(1-m, 0)$ is non-hyperbolic if and only if $\left(k_{1}, b\right)$ lies on the lines:

$$
l_{1}:\left\{\left(k_{1}, b\right) \mid k_{1}=\frac{(1-m) \beta}{(1-m) \beta+2}, 0<b<1\right\}
$$

And

$$
l_{2}:\left\{\left(k_{1}, b\right) \mid k_{1}=1,0<b<1\right\} .
$$

Otherwise, the equilibrium point $P(1-m, 0)$ is an one of the following types: (See Table 1).

Proof. The Jacobian matrix of map (5) at $P(1-m, 0)$ is:

$$
D F(P)=\left[\begin{array}{cc}
1-b & -(1-m)(\beta+b-p d) \\
0 & \beta(1-m)+1-(p d+r)
\end{array}\right]
$$

And its eigenvalues are

$$
\lambda_{1}=1-b, \quad \lambda_{2}=1+(1-m) \beta\left(1-\frac{1}{k_{1}}\right) .
$$

From the assumption $0<b<1$, we see that $0<\lambda_{1}<1$. Then non-hyperbolic will be happened in the case $\lambda_{2}= \pm 1$. From $\lambda_{2}=1$ and $0<m<1$, we get that $k_{1}=1$ and $\left(k_{1}, b\right)$ lies on $l_{2}$. Also, from $\lambda_{2}=-1$, we know $k_{1}=\frac{(1-m) \beta}{(1-m) \beta+2}$ which means $\left(k_{1}, b\right)$ lies on $l_{1}$. When $0<k_{1}<\frac{(1-m) \beta}{(1-m) \beta+2}$ (referred to the case $\left.D_{1}\right)$, the eigenvalue $\lambda_{2}$ satisfies $\lambda_{2}<-1$, then the equilibrium point $P$ is a saddle. When $\frac{(1-m) \beta}{(1-m) \beta+2}<k_{1}<1 \quad\left(\right.$ referred to the case $D_{2}$ ), the eigenvalue $\lambda_{2}$ satisfie $-1<\lambda_{2}<1$, so the equilibrium point $P$ is a stable node and meanwhile when $k_{1}>1$ (referred to the case $D_{3}$ ), the equilibrium point $\mathrm{P}$ is a saddle since $\lambda_{2}>1$. The proof is complete.

Theorem 2. We select $s, r$ as parameters. There does not exist non-hyperbolic case for the equilibrium $Q\left(S^{*}, I^{*}\right)$. But the hyperbolicity can be divided into the following cases (I), (II).

(I) When $b<\beta$, there exist six types for hyperbolic equilibrium point $Q$ : (See Table 2).

Table 1. Types of hyperbolic equilibrium point $P(1-m, 0)$.

\begin{tabular}{cccc}
\hline Cases & Conditions & Eigenvalues & Properties \\
\hline$D_{1}$ & $0<k_{1}<\frac{(1-m) \beta}{(1-m) \beta+2}$ & $0<\lambda_{1}<1, \lambda_{2}<-1$ & saddle \\
$D_{2}$ & $\frac{(1-m) \beta}{(1-m) \beta+2}<k_{1}<1$ & $0<\lambda_{1}<1,-1<\lambda_{2}<1$ & stable nod \\
$D_{3}$ & $k_{1}>1$ & $0<\lambda_{1}<1,1<\lambda_{2}$ & saddle \\
\hline
\end{tabular}


Table 2. Types of hyperbolic equilibrium point $Q\left(S^{*}, I^{*}\right)$.

\begin{tabular}{|c|c|c|c|}
\hline Cases & Conditions & Eigenvalues & Properties \\
\hline$C_{1}$ & $r=\frac{(s-b)^{2}+4 m s q b}{4 s}, b_{1} \leq s \leq b$ & $0<\lambda_{1}=\lambda_{2}<1$ & stable node \\
\hline$C_{2}$ & $r=\frac{(s-b)^{2}+4 m s q b}{4 s}, b<s<\beta$ & $0<\lambda_{1}=\lambda_{2}<1$ & stable node \\
\hline$D_{4}$ & $-b<s<0$ & $1<\lambda_{1}, \quad 0<\lambda_{2}<1$ & saddle \\
\hline$D_{5}$ & $0<s<s_{1}$ & $0<\lambda_{1}<1, \quad 0<\lambda_{2}<1$ & stable node \\
\hline$D_{6}$ & $S_{1}<S<S_{2}$ & $\lambda_{1,2}$ are complex $\left|\lambda_{1,2}\right|<1$ & stable focus \\
\hline$D_{7}$ & $S_{2}<s$ & $0<\lambda_{1}<1, \quad 0<\lambda_{2}<1$ & stable node \\
\hline
\end{tabular}

Where $s_{1}, s_{2}$ satisfy

$$
\begin{gathered}
\left(s_{1}, r\right) \in C_{1}:\left\{\left(s_{1}, r\right) \mid r=\frac{\left(s_{1}-b\right)^{2}+4 m s_{1} q b}{4 s_{1}}, b_{1}=[\sqrt{(b+1)}-1]^{2} \leq s_{1} \leq b\right\} \\
\left(s_{2}, r\right) \in C_{2}:\left\{\left(s_{2}, r\right) \mid r=\frac{\left(s_{2}-b\right)^{2}+4 m s_{2} q b}{4 s_{2}}, b<s_{2}<\beta\right\}
\end{gathered}
$$

respectively.

(II) When $b_{1}<\beta \leq b$, there exist four types for hyperbolic equilibrium point $Q$ : (See Table 3).

Where $s_{3}$ satisfies $\left(s_{3}, r\right) \in C_{3}:\left\{\left(s_{3}, r\right) \mid r=\frac{\left(s_{3}-b\right)^{2}+4 m s_{3} q b}{4 s_{3}}, b_{1}<s_{3}<\beta\right\}$.

Proof. Performing a coordinate shift as follows:

$$
\tilde{S}=S-S^{*}, \tilde{I}=I-I^{*}
$$

and letting $\tilde{F}$ denote the transformed $F$, we translate equilibrium $Q\left(S^{*}, I^{*}\right)$ into $Q_{0}=(0,0)$ and discuss equilibrium point $Q_{0}=(0,0)$ of the map $\tilde{F}$. The matrix of linearization of $\tilde{F}$ at $Q_{0}=(0,0)$ is

$$
\begin{aligned}
D \tilde{F}((0,0)) & =\left[\begin{array}{cc}
1-b-\frac{b[(1-m) \beta-p d-r]}{(1-m) b+r+m p d} & -b-r+m b-m p d \\
\frac{b[(1-m) \beta-p d-r]}{(1-m) b+r+m p d} & 1
\end{array}\right] \\
& =\left[\begin{array}{cc}
a-s & a-r-1+m q(1-a) \\
s & 1
\end{array}\right]
\end{aligned}
$$

where $a=1-b, \quad s=\frac{b[(1-m) \beta-p d-r]}{(1-m) b+r+m p d}$. Its eigenvalues are

$$
\lambda_{1}=\frac{(1+a-s)+\sqrt{(1+a-s)^{2}-4[a+(r-a) s]+4 m s q(1-a)}}{2}
$$


Table 3. Types of hyperbolic equilibrium $Q\left(S^{*}, I^{*}\right)$.

\begin{tabular}{cccc}
\hline Cases & Conditions & Eigenvalues & Properties \\
\hline$C_{3}$ & $r=\frac{(s-b)^{2}+4 m s q b}{4 s}, b \leq s<\beta$ & $0<\lambda_{1}=\lambda_{2}<1$ & stable node \\
$D_{8}$ & $-b<s<0$ & $1<\lambda_{1}, 0<\lambda_{2}<1$ & saddle \\
$D_{9}$ & $0<s<s_{3}$ & $0<\lambda_{1}<1,0<\lambda_{2}<1$ & stable node \\
$D_{10}$ & $s_{3}<s<\beta$ & $\lambda_{1,2}$ are complex $\left|\lambda_{1,2}\right|<1$ & stable focus \\
\hline
\end{tabular}

$$
\lambda_{2}=\frac{(1+a-s)-\sqrt{(1+a-s)^{2}-4[a+(r-a) s]+4 m s q(1-a)}}{2} .
$$

It is known that $(0,0)$ is hyperbolic if and only if none of eigenvalues $\lambda_{1}, \lambda_{2}$ lies on the unit circle $S^{1}$. In the following we discuss the eigenvalues in two case, i.e., $b<\beta$ and $b_{1}<\beta \leq b$.

(I) $b<\beta$

When discriminant $\Delta=(1+a-s)^{2}-4[a+(r-a) s]+4 m s q(1-a) \geq 0$, then $\lambda_{1}$ and $\lambda_{2}$ are both real. Because non-hyperbolicity happens if and only if $\left|\lambda_{1}\right|=1$ or $\left|\lambda_{2}\right|=1$. For whether $\lambda_{1}=1$ or $\lambda_{2}=1$, we can get $a-r+m q(1-a)=1$. By condition $a-r+m q(1-a)=1-b-r+m q b=1-r+(m q-1) b$ and $0<b, m, q, r<1$, we see that $m q-1<0$. This is a contradiction with $1-r+(m q-1) b<1$ and $a-r+m q(1-a)=1$, so $\lambda_{1}=1$ and $\lambda_{2}=1$ are impossible. Next, let's examine $\lambda_{1}=-1$ and $\lambda_{2}=-1$. From whether $\lambda_{1}=-1$ or $\lambda_{2}=-1$, we can get $r=(a+1)\left(1-\frac{2}{s}\right)+m q(1-a)$, By condition $0<a<1,-b<s<\beta$, we see that $(a+1)\left(1-\frac{2}{s}\right)>1, m q(1-a)>0$, This is a contradiction with $r<1$, so $\lambda_{1}=-1$ and $\lambda_{2}=-1$ are impossible.

When $\Delta<0, \lambda_{1}$ and $\lambda_{2}$ are a pair of conjugate complex. Since

$$
\begin{aligned}
\left|\lambda_{1}\right|^{2} & =\left|\lambda_{2}\right|^{2}=\frac{1}{4}\left[(1+a-s)^{2}+4(a+(r-a) s)-4 m s q(1-a)-(1-s+a)^{2}\right] \\
& =a+(r-a) s-m s q(1-a)=[(1-m q) s-1] b+(r-1) s+1<1 .
\end{aligned}
$$

Therefore, $\lambda_{1}$ and $\lambda_{2}$ lie inside of $S^{1}$ and the equilibrium point $Q$ is a stable focus referred to the case $\left(D_{6}\right)$.

When $\Delta \geq 0$, the equilibrium point $Q$ Is hyperbolic. If $\Delta=0$, i.e.

$$
4 r s=(a+s-1)^{2}+4 m s q(1-a) .
$$

The matrix has a double real eigenvalue $\lambda_{1}=\lambda_{2}=(1+a-s) / 2$. From the constraint condition $-b<s<\beta$, it is obvious that $0<\lambda_{1}=\lambda_{2}<1$. Therefore, equilibrium point $Q$ is stable node in the case of $C_{1}$ and $C_{2}$.

If $\Delta>0$, i.e., $4 r s<(a+s-1)^{2}+4 m s q(1-a)$ and $-b<s<\beta$, the eigenvalue $\lambda_{1}$ and $\lambda_{2}$ are different real numbers. We first discuss the case that $-b<s<0$, i.e., $(s, r) \in D_{4}$, In this case we have 


$$
\begin{aligned}
\lim _{r \rightarrow 0^{+}} \lambda_{2} & =\lim _{r \rightarrow 0^{+}} \frac{(1+a-s)-\sqrt{(1+a-s)^{2}-4[a+(r-a) s]+4 m s q(1-a)}}{2} \\
& =\frac{(1+a-s)-\sqrt{(1-a-s)^{2}+4 m s q(1-a)}}{2} \\
& =\frac{(2-b-s)-\sqrt{(b-s)^{2}+4 m s q b}}{2} \\
& <\frac{2-b-s}{2}<\frac{2-b-(-b)}{2}=1
\end{aligned}
$$

and

$$
\frac{\mathrm{d} \lambda_{2}}{\mathrm{~d} r}=\frac{s}{\sqrt{(1+a-s)^{2}-4(a+(r-a) s)+4 m s q(1-a)}}<0 .
$$

We have $0<\lambda_{2}<1$ for $(s, r) \in D_{4}$, On the other hand, there also exists $\lambda_{1}>1$ for $(s, r) \in D_{4}$. In fact, since

$$
\begin{aligned}
\lim _{r \rightarrow 0^{+}} \lambda_{1} & =\lim _{r \rightarrow 0^{+}} \frac{(1+a-s)-\sqrt{(1+a-s)^{2}-4[a+(r-a) s]+4 m s q(1-a)}}{2} \\
& =\frac{(1+a-s)-\sqrt{(1-a-s)^{2}+4 m s q(1-a)}}{2} \\
& =\frac{(2-b-s)-\sqrt{(b-s)^{2}+4 m s q b}}{2} \\
& <\frac{2-b-s}{2}<\frac{2-b-(-b)}{2}=1
\end{aligned}
$$

and

$$
\frac{\mathrm{d} \lambda_{1}}{\mathrm{~d} r}=\frac{-s}{\sqrt{(1+a-s)^{2}-4(a+(r-a) s)+4 m s q(1-a)}}>0 .
$$

We have $\lambda_{1}>1,0<\lambda_{2}<1$. Therefore, the equilibrium $Q$ is a stable node as $(s, r) \in D_{4}$.

For the case $0<s<s_{1}<b$, i.e., $(s, r) \in D_{5}$, we have $\frac{\mathrm{d} \lambda_{2}}{\mathrm{~d} r}>0$ and

$$
\lim _{r \rightarrow 0^{+}} \lambda_{2}=\frac{(1+a-s)-\sqrt{(1-a-s)+4 m s q(1-a)}}{2}<\frac{1+a-s}{2}=\frac{2-(b+s)}{2}<1
$$

and

$$
\begin{gathered}
\frac{\mathrm{d} \lambda_{1}}{\mathrm{~d} r}<0, \\
\lim _{r \rightarrow 0^{+}} \lambda_{1}=\lim _{r \rightarrow 0^{+}} \frac{(1+a-s)+\sqrt{(1+a-s)^{2}-4[a+(r-a) s]+4 m s q(1-a)}}{2} \\
=\frac{(2-b-s)+\sqrt{(b-s)^{2}+4 m s q b}}{2}=1-\frac{b+s-\sqrt{(b-s)^{2}+4 m s q b}}{2}
\end{gathered}
$$


$(b+s)^{2} \leq(b-s)^{2}+4 m s q b$, i.e., $m q \geq 1$ and by condition $0<m, q<1$. This is a contradiction with $m q \leq 1$ and $m q \geq 1$. So $b+s \leq \sqrt{(b-s)^{2}+4 m q s b}$ are impossible, i.e., $b+s>\sqrt{(b-s)^{2}+4 m q s b}$. Therefore, we have $0<\lambda_{2}<\lambda_{1}<1$. Therefore, the equilibrium $Q$ is a stable node as $(s, r) \in D_{5}$.

Finally, we study the case of $s_{2}<s,(s, r) \in D_{7}$. We have

$$
\begin{gathered}
\frac{\mathrm{d} \lambda_{1}}{\mathrm{~d} r}=\frac{-s}{\sqrt{(1+a-s)^{2}-4(a+(r-a) s)+4 m s q(1-a)}}<0, \\
\lim _{r \rightarrow 0^{+}} \lambda_{1}=\lim _{r \rightarrow 0^{+}} \frac{(1+a-s)+\sqrt{(1+a-s)^{2}-4[a+(r-a) s]+4 m s q(1-a)}}{2} \\
=1-\frac{b+s-\sqrt{(b-s)^{2}+4 m s q b}}{2}<1
\end{gathered}
$$

Then, we have $0<\lambda_{1}<1$ for $(s, r) \in D_{7}$. Moreover, there also has $0<\lambda_{2}<1$ for $(s, r) \in D_{7}$. In fact that,

$$
\begin{gathered}
\frac{\mathrm{d} \lambda_{2}}{\mathrm{~d} r}=\frac{s}{\sqrt{(1-s+a)^{2}-4(a+(r-a) s)+4 m s q(1-a)}}>0 \\
\lim _{r \rightarrow 0^{+}} \lambda_{2}=\frac{(1+a-s)-\sqrt{(1-a-s)^{2}+4 m s q(1-a)}}{2}>\frac{(1+a-s)-\sqrt{(1-a-s)^{2}}}{2}=a>0
\end{gathered}
$$

and

$$
\lim _{r \rightarrow 0^{+}} \lambda_{2}=\frac{(1+a-s)-\sqrt{(1-a-s)+4 m s q(1-a)}}{2}<1
$$

We have $0<\lambda_{2}<\lambda_{1}<1$. This means that the equilibrium $Q$ is a stable node for $(s, r) \in D_{7}$.

(II) $b_{1}<\beta \leq b$

When discriminant $\Delta \geq 0$, because non-hyperbolicity happens if and only if $\left|\lambda_{1}\right|=1$ or $\left|\lambda_{2}\right|=1$. Similar to the proof in case (I), neither $\lambda_{1}=1, \lambda_{2}=1$ nor $\lambda_{1}=-1, \lambda_{2}=-1$ is possible.

When $\Delta<0, \lambda_{1}$ and $\lambda_{2}$ are a pair of conjugate complex. Since

$$
\begin{aligned}
\left|\lambda_{1}\right|^{2} & =\left|\lambda_{2}\right|^{2}=\frac{1}{4}\left[(1+a-s)^{2}+4(a+(r-a) s)-4 m s q(1-a)-(1-s+a)^{2}\right] \\
& =a+(r-a) s-m s q(1-a)=[(1-m q) s-1] b+(r-1) s+1<1 .
\end{aligned}
$$

Therefore, $\lambda_{1}$ and $\lambda_{2}$ lie inside of $S^{1}$ and the equilibrium point $Q$ is a stable node referred to the case $\left(D_{10}\right)$.

When $\Delta \geq 0$, the equilibrium point $Q$ is hyperbolic. If $\Delta=0$, the matrix has a double real eigenvalue $\lambda_{1}=\lambda_{2}=\frac{1+a-s}{2}$. From the constraint condition $-b<s<\beta$, it is obvious that $0<\lambda_{1}=\lambda_{2}<1$. Therefore, equilibrium point $Q$ is stable node in the case of $C_{3}$. If $\Delta>0$, we first discuss the case that $-b<s<0$, i.e., $(s, r) \in D_{8}$, In this case we have 


$$
\frac{\mathrm{d} \lambda_{2}}{\mathrm{~d} r}<0, \lim _{r \rightarrow 0^{+}} \lambda_{2}<1
$$

We have $0<\lambda_{2}<1$ for $(s, r) \in D_{8}$, On the other hand, there also exists $\lambda_{1}>1$ for $(s, r) \in D_{8}$. In fact, since $\frac{\mathrm{d} \lambda_{1}}{\mathrm{~d} r}>0, \lim _{r \rightarrow 0^{+}} \lambda_{1}>1$. Therefore, we have $\lambda_{1}>1,0<\lambda_{2}<1$. Therefore, the equilibrium $Q$ is a saddle as $(s, r) \in D_{8}$.

Finally, we study the case of $0<s<s_{3}$, i.e. $(s, r) \in D_{9}$, We easily prove $0<\lambda_{2}<\lambda_{1}<1$ by same methods as in case (I). This means that the equilibrium $Q$ is a stable node for $(s, r) \in D_{9}$. The proof is complete.

\section{Transcritical Bifurcation of the Model}

The following lemmas were be derived from reference [15].

Lemma 1. ([15], Theorem 2.1.4) The map

$$
\left\{\begin{array}{l}
x \mapsto A x+f(x, y), \\
y \mapsto B y+g(x, y),
\end{array} \quad(x, y) \in R^{m} \times R^{n}\right.
$$

satisfies that $A$ is cxc matrix with eigenvalues of modulus one, and $B$ is sxs matrix with eigenvalues of modulus less than one, and

$$
\left\{\begin{array}{l}
D f(0,0)=f(0,0)=0, \\
D g(0,0)=g(0,0)=0,
\end{array}\right.
$$

where $f$ and $g$ are $C^{r} \quad(r \geq 2)$ in some neighborhood of the origin. Then there exists a $C^{r}$ center manifold for equation (7) which can be locally represented as a graph as follows

$$
W^{m}(0)=\left\{(x, y) \in R^{m} \times R^{n}|y=h(x),| x \mid<\delta, h(0)=0, D h(0)=0\right\}
$$

For $\delta$ sufficiently small. Moreover, the dynamics of equation (4.1) restricted to the center manifold is, for $\mu$ sufficiently small, given by the c-dimensional map

$$
u \mapsto A u+f(u, h(u)), u \in R^{m} .
$$

Lemma 2. ([15], in page 365) $A$ one-parameter family of $C^{r} \quad(r \geq 2)$ one-dimensional maps

$$
x \mapsto f(x, \mu), x \in R^{1}, \mu \in R^{1} .
$$

Having a non-hyperbolic fixed point, i.e.,

$$
f(0,0)=0, \frac{\partial f}{\partial x}(0,0)=1
$$

Undergoes a transcritical bifurcation at $(x, \mu)=(0,0)$ if

$$
\frac{\partial f}{\partial \mu}(0,0)=0, \frac{\partial^{2} f}{\partial x \partial \mu}(0,0) \neq 0, \frac{\partial^{2} f}{\partial x^{2}}(0,0) \neq 0
$$

Theorem 3. A transcritical bifurcation occurs at the equilibrium $P(0,0)$ when $k_{1}=1$. More concretely, for $k_{1}<1$ slightly there are two equilibriums: a stable point $P$ 
and an unstable negative equilibrium which coalesce at $k_{1}=1$, for $k_{1}>1$ slightly there are also two equilibriums: an unstable equilibrium $P$ and a stable positive equilibrium $Q$. Thus an exchange of stability has occurred at $k_{1}=1$.

Proof. For $\left(k_{1}, b\right) \in l_{2}$, we have $\lambda_{2}=1$ and $0<\lambda_{1}<1$. Consider $k_{1}$ as the bifurcation parameter and write $F$ as $F_{k_{1}}$ to emphasize the dependence on $\omega$. Performing a coordinate shift as follows $\tilde{S}=S-(1-m), \quad \tilde{I}=I$. One can easily see that the matrix $D F_{k_{1}}(0,0)$ is

$$
\mathbf{D F}_{k_{1}}(0,0)=\left[\begin{array}{cc}
1-b & -(p d+r) k_{1}-(1-m)(b-p d) \\
0 & (p d+r)\left(k_{1}-1\right)+1
\end{array}\right]
$$

and it has eigenvectors

$$
(1,0)^{\mathrm{T}},\left(1, \frac{-b}{(p d+r) k_{1}+(1-m)(b-p d)}\right)^{\mathrm{T}}
$$

Corresponding to $\lambda_{1}$ and $\lambda_{2}$ respectively, where $T$ means the transpose of matrices. First, we put the matrix $D F_{k_{1}}(0,0)$ into a diagonal form. Using the eigenvectors (9), we obtain the transformation

$$
\left[\begin{array}{l}
S \\
I
\end{array}\right]=\left[\begin{array}{cc}
1 & 1 \\
0 & -\frac{b}{(1-m)(\beta+b-p d)}
\end{array}\right]\left[\begin{array}{l}
u \\
v
\end{array}\right]
$$

with inverse

$$
\left[\begin{array}{l}
u \\
v
\end{array}\right]=\left[\begin{array}{cc}
1 & \frac{(1-m)(\beta+b-p d)}{b} \\
0 & -\frac{(1-m)(\beta+b-p d)}{b}
\end{array}\right]\left[\begin{array}{l}
S \\
I
\end{array}\right]
$$

which transform system Equation (5) into

$$
\left[\begin{array}{l}
u \\
v
\end{array}\right] \mapsto\left[\begin{array}{cc}
1-b & 0 \\
0 & 1
\end{array}\right]\left[\begin{array}{l}
u \\
v
\end{array}\right]+\left[\begin{array}{c}
A \beta u v+A \beta v^{2}+(B+C) v \\
\beta u v+\beta v^{2}-(1-m) \beta v
\end{array}\right]
$$

where

$$
A=\frac{b-(1-m)(\beta+b-p d)}{(1-m)(\beta+b-p d)}, B=p d+r-b, C=\frac{b(b-p d)}{(\beta+b-p d)} .
$$

Rewrite system (12) in the suspended form with assumption $\omega=k_{1}-1$,

$$
\left[\begin{array}{c}
u \\
v \\
\omega
\end{array}\right] \mapsto\left[\begin{array}{ccc}
1-b & 0 & 0 \\
0 & 1 & 0 \\
0 & 0 & 1
\end{array}\right]\left[\begin{array}{c}
u \\
v \\
\omega
\end{array}\right]+\left[\begin{array}{c}
a_{11} u v+a_{12} v^{2}+a_{13} v \omega \\
a_{21} u v+a_{22} v^{2}+a_{23} v \omega \\
0
\end{array}\right]
$$

where

$$
a_{11}=a_{12}=A \beta, a_{21}=a_{22}=\beta, a_{13}=\frac{(B+C)(p b+r)}{b-(1-m)(\beta+b-p d)}, a_{23}=-\frac{(1-m) \beta}{\beta-p b-r} .
$$

Thus, from Lemma 1 , the stability of equilibrium $(u, v)=(0,0)$. near $k_{1}=1$ can be 
determined by studying an one parameter family of map on a center manifold which can be represented as follows,

$$
\mathrm{W}^{c}(0,0)=\left\{(u, v, \omega) \in R^{3} \mid u=h(v, \omega), h(0,0)=0, D h(0,0)=0\right\} .
$$

for sufficiently small $v$ and $\omega$.

We now want to compute the center manifold and derive the mapping on the center manifold. We assume

$$
h(v, \omega)=d v^{2}+e v \omega+f \omega^{2}+O(3)
$$

near the origin, where $O(3)$ means terms of order $\geq 3$. By Lemma 1, those coefficients $d, e, f$ can be determined by the equation

$$
\begin{aligned}
N(h(v, \omega))= & h\left(v+a_{21} h(v, \omega) v+a_{22} v^{2}+a_{23} v \omega, \omega\right) \\
& -\left[(1-b) h(v, \omega)+a_{11} h(v, \omega) v+a_{12} v^{2}+a_{13} v \omega\right]=0 .
\end{aligned}
$$

Substituting (16)into (15) and comparing coefficients of $v^{2}, v \omega$ and $\omega^{2}$ in (15), we get

$$
\left\{\begin{array}{l}
d+d(b-1)-a_{22}=0 \\
e+e(b-1)-a_{23}=0 \\
f+f(b-1)=0
\end{array}\right.
$$

from which we solve

$$
d=\frac{a_{22}}{b}, e=\frac{a_{23}}{b}, f=0 .
$$

Therefore, the expression of (15) is approximately determined:

$$
h(v, \omega)=\frac{a_{22}}{b} v^{2}+\frac{a_{23}}{b} v \omega+O(3) .
$$

Substituting (17) into (14), we obtain a one dimensional map reduced to the center manifold

$$
v \mapsto \varphi_{\omega}(v)=v+a_{22} v^{2}+a_{23} v \omega+\frac{a_{21} a_{22}}{b} v^{3}+\frac{a_{21} a_{23}}{b} v^{2} \omega+O(4) .
$$

It is easy to check that

$$
\frac{\partial \varphi_{\omega}}{\partial \omega}(0,0)=0, \frac{\partial^{2} \varphi_{\omega}}{\partial v \partial \omega}(0,0) \neq 0, \frac{\partial^{2} \varphi_{\omega}}{\partial v^{2}}(0,0) \neq 0 .
$$

The condition (19) implies that in the study of the orbit structure near the bifurcation point terms of $O(3)$ do not qualitatively affect the nature of the bifurcation, namely they do not affect the geometry of the curves of equilibriums passing through the bifurcation point. Thus, the orbit structure of $(18)$ near $(u, v)=(0,0)$ is qualitatively the same as the orbit structure near $(u, v)=(0,0)$ of the map

$$
v \mapsto v+a_{22} v^{2}+a_{21} v \omega .
$$

Map (20) can be viewed as truncated normal form for the transcritical bifurcation (see Lemma 2). The stability of the two branches of equilibriums lying on both sides of 
$k_{1}=1$ are easily verified.

\section{Degenerate Flip Bifurcation of the Model}

This section is devoted to the analysis for the case $\left(k_{1}, b\right) \in l_{1}$. From section 2 , we have $\lambda_{2}=-1, \lambda_{1}=1-b, k_{1}=\frac{(1-m) \beta}{(1-m) \beta+2}$ for $\left(k_{1}, b\right) \in l_{1}$. For this case, degenerate flip bifurcation happens at the equilibrium point $P(1-m, 0)$.

Theorem 4. For map (5) when $\left(k_{1}, b\right) \in l_{1}$, degenerate flip bifurcation happens at the equilibrium point $P(1-m, 0)$.

Proof. Performing a coordinate shift as follows

$$
\tilde{S}=S-(1-m), \quad \tilde{I}=I,
$$

We translate equilibrium $P(1-m, 0)$ into $(0,0)$, and letting $\tilde{F}$ denote the transformed $F$

$$
\left[\begin{array}{c}
\tilde{S} \\
\tilde{I}
\end{array}\right] \mapsto\left[\begin{array}{c}
-\beta \tilde{S} \tilde{I}+(1-b) \tilde{S}-(1-m)(b+\beta-p d) \tilde{I} \\
\beta \tilde{S} \tilde{I}+(\beta(1-m)+1-(p d+r)) \tilde{I}
\end{array}\right]
$$

Therefore, we discuss equilibrium point $(0,0)$ of the map $\tilde{F}$. The matrix of linearization of $\tilde{F}$ at $(0,0)$ is

$$
D \tilde{F}((0,0))=\left[\begin{array}{cc}
1-b & -(1-m)(b+\beta-p d) \\
0 & \beta(1-m)+1-(p d+r)
\end{array}\right] .
$$

For $\left(k_{1}, b\right) \in l_{1}$, considering $\omega=k_{1}-1$ as the bifurcation parameter and write $\tilde{F}$ as $\tilde{F}_{\omega}$ to emphasize the dependence on $w$. Therefore, we have

$$
D \tilde{F}_{\omega}((0,0))=\left[\begin{array}{cc}
1-b & -(1-m)(b+\beta-p d) \\
0 & -1
\end{array}\right]
$$

The matrix have eigenvectors $(1,0)^{\mathrm{T}}$ and $\left(1, \frac{2-b}{(1-m)(\beta+b-p d)}\right)^{\mathrm{T}}$ corresponding to $\lambda_{1}=1-b$ and $\lambda_{2}=-1$. Therefore, by transformation

$$
(\tilde{S}, \tilde{I})^{\mathrm{T}}=H(u, v)^{\mathrm{T}}
$$

where

$$
H=\left[\begin{array}{cc}
1 & 1 \\
0 & \frac{2-b}{(1-m)(\beta+b-p d)}
\end{array}\right] .
$$

Therefore, we obtain the inverse of transformation (23)

$$
\left[\begin{array}{l}
u \\
v
\end{array}\right] \mapsto\left[\begin{array}{cc}
1 & \frac{(1-m)(\beta+b-p d)}{2-b} \\
0 & -\frac{(1-m)(\beta+b-p d)}{2-b}
\end{array}\right]\left[\begin{array}{c}
\tilde{S} \\
\tilde{I}
\end{array}\right]
$$

Therefore $\tilde{F}_{\omega}$ can be changed into the maps: $\Phi_{\omega}: R^{2} \rightarrow R^{2}$ 


$$
\left[\begin{array}{l}
u \\
v
\end{array}\right]=\left[\begin{array}{cc}
1-b & 0 \\
0 & -1
\end{array}\right]\left[\begin{array}{l}
u \\
v
\end{array}\right]+\left[\begin{array}{c}
B v-A \beta u v+A \beta v^{2} \\
-(1-m) \beta v+\beta u v+\beta v^{2}
\end{array}\right]
$$

where

$$
A=1+\frac{2-b}{(1-m)(\beta+b-p d)}, \quad B=\left[(p d+r)-b-\frac{(2-b)(b-p d)}{(p+b-p d)}\right] .
$$

Rewrite system (25) in the suspended form

$$
\left[\begin{array}{c}
u \\
v \\
\omega
\end{array}\right]=\left[\begin{array}{ccc}
1-b & 0 & 0 \\
0 & -1 & 0 \\
0 & 0 & 1
\end{array}\right]\left[\begin{array}{l}
u \\
v \\
\omega
\end{array}\right]+\left[\begin{array}{c}
e_{11} v \omega+e_{12} u v+e_{13} v^{2} \\
e_{21} v \omega+e_{22} u v+e_{23} v^{2} \\
0
\end{array}\right]
$$

where

$$
\begin{gathered}
e_{11}=\frac{B}{\beta(1-m)-(p d+r)+2}, \quad e_{21}=\frac{-\beta(1-m)}{\beta(1-m)-(p d+r)+2}, \\
e_{22}=e_{23}=\beta, \quad e_{12}=e_{13}=A \beta .
\end{gathered}
$$

Equivalently, the suspended system (26) has a two-dimensional center manifold of the form

$$
v=h(u, \omega)=d u^{2}+e u \omega+f \omega^{2}+O(3)
$$

Near the origin, where $O(3)$ means terms of order $\geq 3$. By Lemma 1, those coefficients $d, e, f$ can be determined by the equation

$$
\begin{aligned}
N(h(u, \omega)): & =h\left((1-b) u+e_{11} v \omega+e_{12} u v+e_{13} v^{2}, \omega\right) \\
& -\left[-h(u, \omega)+e_{21} \omega h(u, \omega)+e_{22} u h(u, \omega)+e_{23}(h(u, \omega))^{2}\right]=0 .
\end{aligned}
$$

Then

$$
\begin{aligned}
N(h(u, \omega)) & :=d(1-b)^{2} u^{2}+e(1-b) u \omega+f \omega^{2}+d u^{2}+e u \omega+f \omega^{2}+O(3) \\
& =d\left[(1-b)^{2}+1\right] u^{2}+e(2-b) u \omega+2 f \omega^{2}+O(3)=0
\end{aligned}
$$

Comparing coefficients of $u^{2}, u \omega$ and $\omega^{2}$ in (27), we get

$$
\left\{\begin{array}{l}
d\left[(1-b)^{2}+1\right]=0 \\
e(2-b)=0 \\
2 f=0
\end{array}\right.
$$

from which we solve

$$
d=0, e=0, f=0,
$$

Thus, the expression of (27)is determined, i.e.,

$$
v=h(u, \omega)=0+O(3) .
$$

Substituting (30) into the first equation in (26), we obtain a one-dimensional map $u \mapsto \varphi_{\omega}(u)$, where 


$$
\begin{aligned}
\varphi_{\omega}(u) & =(1-b) u+e_{11} \omega h(u, \omega)+e_{12} u h(u, \omega)+e_{13}(h(u, \omega))^{2} \\
& =(1-b) u+e_{11} \omega \times 0+e_{12} u \times 0+e_{13} \times 0^{2} \\
& =(1-b) u .
\end{aligned}
$$

From (31), we can check that

$$
\begin{aligned}
& {\left[\frac{\partial \phi_{\omega}}{\partial \omega} \frac{\partial^{2} \phi_{\omega}}{\partial u^{2}}+2 \frac{\partial^{2} \phi_{\omega}}{\partial u \partial \omega}\right]_{(u, \omega)=(0, \omega)}=0} \\
& {\left[\frac{1}{4}\left(\frac{\partial^{2} \varphi_{\omega}}{\partial u^{2}}\right)^{2}+\frac{1}{6} \frac{\partial^{3} \varphi_{\omega}}{\partial u^{3}}\right]_{(u, \omega)=(0, \omega)}=0 .}
\end{aligned}
$$

Thus, the conditions $\left(F_{1}\right)$ and $\left(F_{2}\right)$ of Theorem 3.5.1 in [16] are not satisfied. Therefore, this is a degenerate flip bifurcation.

\section{Conclusion}

Due to a lot of discrete-time models are not trivial analogues of their continuous ones and simple discrete-time models can even exhibit complex behavior (see [14]), motivated mainly by Meng and Chen [13] considering a class of continuous vertical and horizontal transmitted epidemic model (1) under constant vaccination, we study a class of discrete vertical and horizontal transmitted disease model (2) under constant vaccination. By detailed studies, we found discrete model (2) has a flip bifurcation which did not occurred for continuous model. However, the result of flip bifurcation in current paper is a degenerate situation, for which the more in-depth research needs to be continued.

\section{Acknowledgements}

This work has been supported by the Innovation and Developing School Project of Department of Education of Guangdong province (Grant No. 2014KZDXM065) and the Key project of Science and Technology Innovation of Guangdong College Students (Grant No. pdjh2016a0301).

\section{References}

[1] Piyawong, W., Twizell, E.H. and Gumel, A.B. (2003) An Unconditionally Convergent Finite-Difference Scheme for the SIR Model. Applied Mathematics and Computation, 146, 611-625. https:/doi.org/10.1016/S0096-3003(02)00607-0

[2] Pourabbas, E., d'Onofrio, A. and Rafanelli, M. (2001) A Method to Estimate the Incidence of Communicable Diseases under Seasonal Fluctuations with Application to Cholera. Applied Mathematics and Computation, 118, 161-174. https:/doi.org/10.1016/S0096-3003(99)00212-X

[3] Beretta, E. and Takeuchi, Y. (1997) Convergence Results in SIR Epidemic Model with Varying Population Sizes. Nonlinear Analysis, 28, 1909-1921. https:/doi.org/10.1016/S0362-546X(96)00035-1

[4] Meng, X., Chen, L. and Song, Z. (2007) The Global Dynamics Behaviors for a New Delay SEIR Epidemic Disease Model with Vertical Transmission and Pulse Vaccination. Applied 
Mathematics and Computation, 28, 1259-1271. https:/doi.org/10.1007/s10483-007-0914-x

[5] Allen, L.J.S. (1994) Some Discrete-Time SI, SIR and SIS Epidemic Models. Mathematical Biosciences, 124, 83-105. https:/doi.org/10.1016/0025-5564(94)90025-6

[6] Ma, Z. Zhou, Y. Wang, W. and Jin, Z. (2004) Mathematical Modelling and Research of Epidemic Dynamical Systems (in Chinese). Science Press, Beijing.

[7] Zhou, X., Li, X. and Wang, W.S. (2014) Bifurcations for a Deterministic SIR Epidemic Model in Discrete Time. Advances in Difference Equations, 168.

https:/doi.org/10.1186/1687-1847-2014-168

[8] Liao, X., Wang, H., Huang, X., Zeng, W. and Zhou, X. (2015) The Dynamic Properties of a Deterministic SIR Epidemic Model in Discrete Time. Applied Mathematics, 6, 1665-1675. https:/doi.org/10.4236/am.2015.610148

[9] Meng, X., Chen, L. and Cheng, H. (2007) Two Profitless Delays for the SEIRS Epidemic Disease Model with Nonlinear Incidence and Pulse Vaccination. Applied Mathematics and Computation, 186, 516-529. https:/doi.org/10.1016/j.amc.2006.07.124

[10] Agur, Z.L., et al. (1993) Pulse Mass Measles Vaccination across Age Cohorts. Proceedings of the National Academy of Sciences of the USA, 90, 11698-11702. https:/doi.org/10.1073/pnas.90.24.11698

[11] Shulgin, B., et al. (1998) Pulse Vaccination Strategy in the SIR Epidemic Model. Bulletin of Mathematical Biology, 60, 1-26. https:/doi.org/10.1016/S0092-8240(98)90005-2

[12] Meng, X., Chen, L. and Song, Z. (2007) The Global Dynamics Behaviors for a New Delay SEIR Epidemic Disease Model with Vertical Transmission and Pulse Vaccination. Applied Mathematics and Mechanics (English Edition), 28, 1259-1271. https:/doi.org/10.1007/s10483-007-0914-x

[13] Meng, X. and Chen, L. (2008) The Dynamics of a New SIR Epidemic Model Concerning Pulse Vaccination Strategy. Applied Mathematics and Computation, 197, 582-597. https:/doi.org/10.1016/j.amc.2007.07.083

[14] Anderson, R.M. and May, R.M. (1991) Infections Diseases of Humans: Dynamics and Control. Oxford University Press, Oxford.

[15] Wiggins, S. (1990) Introduction to Applied Nonlinear Dynamical Systems and Chaos. Springer, New York. https:/doi.org/10.1007/978-1-4757-4067-7

[16] Guckenheimer, J. and Holmes, P. (1983) Nonlinear Oscillations, Dynamical Systems and Bifurcations of Vector Fields. Springer, New York.

https:/doi.org/10.1007/978-1-4612-1140-2 
Submit or recommend next manuscript to SCIRP and we will provide best service for you:

Accepting pre-submission inquiries through Email, Facebook, LinkedIn, Twitter, etc. A wide selection of journals (inclusive of 9 subjects, more than 200 journals)

Providing 24-hour high-quality service

User-friendly online submission system

Fair and swift peer-review system

Efficient typesetting and proofreading procedure

Display of the result of downloads and visits, as well as the number of cited articles

Maximum dissemination of your research work

Submit your manuscript at: http://papersubmission.scirp.org/

Or contact ijmnta@scirp.org 